Supplementary Information for:

\title{
Hydrophilic and Double Hydrophilic/Hydrophobic Microcapsules using a Single, Thermally Responsive, Self-Sorting Dispersant
}

Lérys Granado, ${ }^{[a][b]}$ Céline Burel, ${ }^{[b]}$ Rémi Giordanengo, ${ }^{[b]}$ Ahmed Alsayed, ${ }^{[b]}$ Denis Bendejacq ${ }^{[b]}$ and François Ganachaud ${ }^{*[a][b]}$

[a] INSA-Lyon, Ingénierie des Matériaux Polymères, 69621 Villeurbanne (France)

and

CNRS, UMR 5223, Ingénierie des Matériaux Polymères, 69621 Villeurbanne (France)

[b] Complex Assemblies of Soft Matter, UMI 3254, Solvay/CNRS/Upenn,

350 George Patterson Boulevard, Bristol, Pennsylvania, 19007, United States

*Corresponding author: françois.ganachaud@insa-lyon.fr

\section{Table of Contents}

SI.1: Experimental part $\quad 2$

SI.2: Preliminary screening of different PVAs 6

SI.3: Determination of PVAl80-10 microstructure by NMR 7

SI.4: Analysis of some PVAl80-10 properties by DSC 10

SI.5: PVAl80-10 crystallinity determined by X-ray diffraction (XRD) 11

SI.6: Determination of PVAl80-10's LCST by DLS 12

SI.7: Water-in-chloroform interfacial tensions 13

SI.8: Histograms of microcapsules $\quad 14$

$\begin{array}{ll}\text { SI.9: Shell thickness of w/w dispersion } & 16\end{array}$

SI.10: Kinetics and optimization of crosslinking reaction 17

SI.11: Calibration curve of methyl blue 18

$\begin{array}{lr}\text { SI.12: Phase diagrams } & 19\end{array}$

SI.13: Images of ethyl acetoacetate-water dispersions 20

SI.14: Movie description 21

References $\quad 22$ 


\section{SI.1: Experimental part \\ Materials}

Poly(vinyl alcohol) (Alcotex) $55 \%$ and $72 \%$ hydrolyzed were kindly provided by Synthomer. Poly(vinyl alcohol)s (9-10 kDa, 80\% hydrolyzed) and (33-60 kDa, 98\% hydrolyzed), sodium sulfate, sodium hydroxide, ethyl acetoacetate, (-)-a-terpineol (biobased), doxorubicin hydrochloride, paclitaxel, deuterated-chloroform, Rhodamine $110 \mathrm{HCl}$, Rhodamine $\mathrm{B}$ isothiocyanate, methyl blue (i.e. acid blue 93), Nile Red and glutaraldehyde ( 50 vol\% in water) were purchased from SigmaAldrich and used as received. Chloroform (stabilized with $500 \mathrm{ppm}$ ethanol), deuterium oxide and hydrochloric acid (37\%) were purchased from VWR. Water was either in-house de-ionized (DI) water or highly pure water $\left(18 \mathrm{M} \Omega \mathrm{cm}^{-1}\right)$.

\section{Methods}

Gel permeation chromatography was performed using a midiDAWN (Wyatt), equipped with a triple detection. The column was a Shodek OHpak $806 \times 3(30 \mu \mathrm{m})$. The mobile phase was $0.1 \mathrm{M} \mathrm{NaNO}_{3}$ (100 ppm $\mathrm{NaN}_{3}$ ) with a flow rate of $1 \mathrm{~mL} / \mathrm{min}$, at room temperature. The apparatus was calibrated using pullulan standards.

Nuclear magnetic resonance (NMR) spectra were acquired on a Bruker AC $400 \mathrm{MHz}$ instrument.

Interfacial tensions between PVA aqueous solution and chloroform were measured on a Tracker apparatus from Teclis Instruments. The chloroform was previously saturated with water. Drops of PVA aqueous solution were inserted into chloroform as the static phase, and the contact angle measurement was performed during $120 \mathrm{~s}$.

The differential scanning calorimetry (DSC) apparatus used here was a DSC Q200 from TA instruments. The calibration was freshly performed with highly pure indium standards, at $10 \mathrm{~K} / \mathrm{min}$. The carrier gas was nitrogen, at a flow of $50 \mathrm{~mL} / \mathrm{min}$. About $8 \mathrm{mg}$ of PVA powder were inserted in aluminum crucibles and sealed with pierced lids.

The X-rays diffraction (XRD) apparatus was a Miniflex from Rigaku, using the Cu Ka emission line with a $\theta-2 \theta$ geometry. The PVA powder and w/o emulsion were deposited on a Si wafer and analyzed as-is.

The dynamic light scattering apparatus was a ZetaSizer Nano from Malvern. Thermostated DLS was used to determine the cloud point of PVAl80-10 in a $0.1 \mathrm{M}$ sodium sulfate aqueous solution at various PVA concentrations (\% weight/volume, w/v). The temperature was increased from 25 to 70 ${ }^{\circ} \mathrm{C}$, with $2{ }^{\circ} \mathrm{C}$ increments (5 minutes equilibration between two increments).

Optical microscopy images were acquired by confocal laser scanning microscopy on a Leica SP8 in bright field and transmission mode for the grey images and in fluorescence mode for the fluorescence images. The microscope was equipped with a Leica 60X oil immersion objective and 
spectroscopic detector. Typical excitation lines and emission wavelengths for the fluorophores of this study were Rhodamine $110 \mathrm{HCl}$ (hydrophilic dye, $\lambda_{e x}=488 \mathrm{~nm}, \lambda_{e m}=500-550 \mathrm{~nm}$ ), Nile Red (hydrophobic dye, $\lambda_{e x}=552 \mathrm{~nm}, \lambda_{e m}=600-800 \mathrm{~nm}$, Rhodamine B isothiocyanate (PVA labelling, hydrophobic, $\lambda_{e x}=552 \mathrm{~nm}, \lambda_{e m}=600-800 \mathrm{~nm}$ ).

Scanning electron microscopy (SEM) was performed on a FEI Quanta 600 ESEM. The samples were prepared by air drying under ambient conditions by laying a drop of PVA microcapsules dispersion on a glass slide with xylene as cosolvent to slow down the drying stage and maintain the capsules morphology. The samples were not metallized prior to investigation.

Emulsification process. Typical emulsification processes were the following (see details in Table S1). For route (1), $10 \mathrm{~mL}$ of chloroform and $10 \mathrm{~mL}$ of aqueous PVA $5 \% \mathrm{w} / \mathrm{v}$ in $0.1 \mathrm{M}$ Sodium sulfate were inserted in a vial and let equilibrate at $50^{\circ} \mathrm{C}$ for $20 \mathrm{~min}$. Then the emulsification was performed using typically an ultra-turrax homogenizer $\left(25000 \mathrm{rpm}, 30 \mathrm{~min}, 50^{\circ} \mathrm{C}\right)$. Alternatively, emulsification can be performed using ultrasonication $\left(1 \mathrm{~min}, 50^{\circ} \mathrm{C}\right)$. Respectively, $30 \mathrm{~min}$ and 1 min were necessary in order to observe no evolution of particle size and gave similar results. For solvent transfer, the w/o emulsion were centrifugated at $3500 \mathrm{rpm}$ for $5 \mathrm{~min}$. The under-natant chloroform was removed, and the excess of solvent was let to evaporate for $30 \mathrm{~min}$. The coacervated capsules were then redispersed in water by adding an aqueous solution.

Table S1. Typical operating conditions of emulsification .

\begin{tabular}{ll}
\hline Conditions & Typical values \\
\hline Water phase & PVA (80\% hydrolyzed, 9.8 kDa) $5 \%$ w/v in $0.1 \mathrm{M} \mathrm{Na}_{2} \mathrm{SO}_{4}$ \\
Oil phase & Chloroform (route 1) or Ethyl Acetoacetate (route 2) \\
& Ultra-Turrax homogenizers, $25000 \mathrm{rpm}, 30 \mathrm{~min}, 50^{\circ} \mathrm{C}$ \\
$\mathrm{W} / \mathrm{O}$ emulsification & Alternatively: ultrasonication during $1 \mathrm{~min}, 50^{\circ} \mathrm{C}$ \\
$\mathrm{O} / \mathrm{W}$ emulsification & Agitation, $400 \mathrm{rpm}, 10 \mathrm{~min}, 25^{\circ} \mathrm{C}$ \\
Volume water:oil & Route 1:50:50; Route 2:1) 20:80, 2) $80: 20$ \\
\hline
\end{tabular}

For route (2), $16 \mathrm{~mL}$ of ethyl acetoacetate and $4 \mathrm{~mL}$ of aqueous PVA $5 \% \mathrm{w} / \mathrm{v}$ in $0.1 \mathrm{M}$ Sodium sulfate were inserted in a vial and let to equilibrate at $50^{\circ} \mathrm{C}$ for $20 \mathrm{~min}$, followed by emulsification using ultra-turrax homogenizer (25000 rpm, $30 \mathrm{~min}, 50^{\circ} \mathrm{C}$ ). To form a w/o/w dispersion, $4 \mathrm{~mL}$ of the previously formed w/o dispersion were dispersed in $16 \mathrm{~mL}$ of $0.1 \mathrm{M}$ Sodium sulfate aqueous solution by magnetic stirring for $10 \mathrm{~min}$ at $25^{\circ} \mathrm{C}$ and $400 \mathrm{rpm}$. To form w/o/w/o dispersions, $4 \mathrm{~mL}$ of the previously formed w/o/w dispersion were redispersed in $16 \mathrm{~mL}$ of ethyl acetoacetate at $50^{\circ} \mathrm{C}$.

PVA labelling for capsules and microscopic observations. PVA was tagged with Rhodamine B isothiocyanate (RBTIC) by reaction between alcohol and isothiocyanate yielding thiourethane. The following method was applied: $30 \mathrm{mg}$ of RBTIC, $5 \mathrm{~g}$ of PVA and $0.1 \mathrm{~mL}$ aqueous $0.1 \mathrm{M}$ sodium hydroxide. were dissolved in DMSO. The mixture was allowed to react during 48 hours at room 
temperature under magnetic agitation. Then, the mixture was dialyzed against milli- $Q$ water (using $5 \mathrm{kDa}$ cutoff membranes) until reaching a transparent dialysis solution. Owing to the fact that RBTIC represents less than $1 \%$ by mass of PVA and that the thioisocyanate-alcohol reaction is not expected to be total, we assumed here that the low functionalization does not modify drastically PVA properties.

To observe the microcapsules, dialyzed tagged-PVA was diluted to obtain $10 \mathrm{~mL}$ of $0.1 \mathrm{M}$ sodium sulfate, tagged-PVA $5 \% \mathrm{w} / \mathrm{v}$ solution. Then, $10 \mathrm{~mL}$ chloroform was added, and the two phases were equilibrated at $50{ }^{\circ} \mathrm{C}$. After $20 \mathrm{~min}$, the emulsification was performed using Ultra-Turrax homogenizer at $5000 \mathrm{rpm}$ during $5 \mathrm{~min}$, rather than at $25000 \mathrm{rpm}, 30 \mathrm{~min}$, to obtain bigger capsules and observe the hollow morphology and shell membrane. After creaming of the emulsion within few minutes, ca. $1 \mathrm{~mL}$ of capsules were re-dispersed in $5 \mathrm{~mL} 0.1 \mathrm{M}$ sodium sulfate aqueous solution for fluorescence confocal microscopy observation. Intensity profiles were plotted for individual capsules using imageJ. The shell wall was characterized by a peak which was well fitted with a Gaussian function. The shell thickness was taken at the full width at half maximum.

Capsules crosslinking. $40 \mathrm{~mL}$ of chloroform and $40 \mathrm{~mL}$ of a PVA $5 \% \mathrm{w} / \mathrm{v} 0.1 \mathrm{M}$ sodium sulfate aqueous solution were inserted in a round-bottom flask. After equilibration at $50{ }^{\circ} \mathrm{C}$ for $30 \mathrm{~min}$, the mixture was emulsified. Then, were inserted $658 \mu \mathrm{L}$ of toluene (as internal reference), $329 \mu \mathrm{L}$ of a glutaraldehyde 50 vol\% solution (i.e. 20 mol\% with respect to the number of moles of PVA hydroxyl groups) and $667 \mu \mathrm{L}$ of hydrochloric acid $12 \mathrm{M}$. The reaction was performed at $50^{\circ} \mathrm{C}$ under magnetic agitation. Regularly, samples of $1.5 \mathrm{~mL}$ were taken. Samples were centrifugated at $10000 \mathrm{rpm}$ for 5 min. The supernatant was analyzed by ${ }^{1} \mathrm{H}$ NMR spectroscopy using $\mathrm{CDCl}_{3}$ solvent.

UV-vis spectroscopy quantification method. Methyl blue (MB) was selected to mimic a hydrophilic active to encapsulate according to its high hydrophilicity, strong UV-vis response, and costeffectiveness. First, an UV-vis spectroscopic quantification method was developed. From an aqueous MB stock solution ( $4.3 \mathrm{mg} / \mathrm{mL}), 20 \mathrm{~mL}$ of standard solutions were prepared by dilution of an aqueous solution of $0.1 \mathrm{M}$ sodium sulfate and $\mathrm{pH}$ was adjusted to 1 using concentrated hydrochloric acid (as MB display a maximum absorbance at $\mathrm{pH} 1$ ). The maximum UV-vis absorbance of MB was found at $600 \mathrm{~nm}$. Calibration curve shows excellent linearity and was used to quantify the amount of MB released from the microcapsules to water continuous phase. One standard was regularly probed to check the accuracy of the calibration curve.

Cumulative cargo release. A typical procedure to assess the MB release was the following. $1 \mathrm{~mL}$ of MB stock solution was homogenized in $10 \mathrm{~mL}$ PVA $5 \% \mathrm{w} / \mathrm{v}$ aqueous solution. $10 \mathrm{~mL}$ chloroform was added. After equilibration during $20 \mathrm{~min}$ at $50^{\circ} \mathrm{C}$, the two-phase mixture was emulsified one minute by ultrasonication. For the cross-linked capsules $(80 \mu \mathrm{L}$ of glutaraldehyde $5 \mathrm{wt} \%$ solution, i.e. 2 mol\% with respect to the total moles of PVA hydroxyl units, $+200 \mu \mathrm{L}$ Hydrochloric acid $12 \mathrm{M}$ ), the crosslinker was allowed to react with PVA during 2 hours at $50^{\circ} \mathrm{C}$ under $500 \mathrm{rpm}$ agitation. Afterward, in any case, the capsule was separated from chloroform using centrifugation at 3500 rpm for 5 min. Residual chloroform was allowed to evaporate from the capsule during $\mathbf{3 0}$ min under 
atmospheric pressure. The capsules were re-dispersed in $0.1 \mathrm{M}$ Sodium sulfate aqueous solution for a total volume of $200 \mathrm{~mL}$ (dilution factor of 5:95 allowed maintaining the same osmotic pressure throughout the release test). The $\mathrm{pH}$ was adjusted to 5.5 using concentrated sodium hydroxide or hydrochloric acid (the pH of PVAl80-10 neat aqueous solutions). The release was performed at 25 ${ }^{\circ} \mathrm{C}$, under magnetic agitation (500 rpm). For each point, 3 samples of $1.5 \mathrm{~mL}$ were taken from the stirred releasing medium (without refill with clear solution), and centrifugated one minute at 10000 $\mathrm{rpm}$. The supernatant free of capsule, i.e. clear, blue-colored solution, was then analyzed in UVvis, after $\mathrm{pH}$ adjustment to 1 using Hydrochloric acid. The cargo released value deviations between the three specimens were negligible $(<1 \%)$.

Dual hydrophilic/hydrophobic anticancer drug encapsulation. $2 \mathrm{mg}$ of doxorubicin hydrochloride (DOX) were dissolved in a PVAl80-10 5\% w/v aqueous solution (DOX-PVA solution). $5 \mathrm{mg}$ of paclitaxel (PTX) were dissolved in $3 \mathrm{~mL}$ of $\alpha$-terpineol (PTX- $\alpha \mathrm{T}$ solution). $200 \mu \mathrm{L}$ of DOX-PVA and $800 \mu \mathrm{L}$ of PAX- $\alpha \mathrm{T}$ were inserted in a vial, heated at $50^{\circ} \mathrm{C}$ for $15 \mathrm{~min}$ and emulsified 1 minute using ultrasonication. Then, after cooling down to room temperature, the w/o dispersion was dispersed in $4 \mathrm{~mL}$ of a $5 \% \mathrm{w} / \mathrm{v}$ PVAl80-10, $0.1 \mathrm{M}$ sodium sulfate aqueous solution yielding after hand mixing a $\mathrm{w} / \mathrm{o} / \mathrm{w}$ dispersion. The fluorescence microscopic observations were subsequently performed on a Zeiss microscope using a blue excitation line $(450-500 \mathrm{~nm})$ and a color CCD camera. DOX-PVA domains appeared in fluorescent green and PTX- $\alpha \mathrm{T}$ ones in red. 


\section{SI.2: Preliminary screening of different PVAs}

Aqueous PVA solutions were prepared by dissolving the different PVAs in DI water and adding the according amount of sodium sulfate to reach $0.1 \mathrm{M} \mathrm{Na}_{2} \mathrm{SO}_{4}$. PVAs with hydrolysis degree of less than $80 \%$ gave cloudy colloidal aqueous solutions, even without salt. Then, emulsions were prepared by mixing $10 \mathrm{~mL}$ PVA solution with $10 \mathrm{~mL}$ chloroform (50:50, v:v), equilibrated $20 \mathrm{~min}$ at the desired temperature and sonicated one minute. Only PVAl80-10 showed the capacity of stabilizing o/w and $\mathrm{w} / \mathrm{o}$ dispersions below and above its LCST, respectively. Therefore, this polymer was selected to carry out this study.

Table S2. Screening on emulsification behavior of different PVAs.

\begin{tabular}{|c|c|c|c|c|c|}
\hline $\begin{array}{c}\text { PVA trade name, } \\
\text { Ref and Supplier }\end{array}$ & Acronym & $\begin{array}{c}\text { Hydrolysis } \\
\text { degree }(\%)\end{array}$ & $\begin{array}{c}\boldsymbol{M}_{\mathbf{w}} \\
\mathbf{( k D a})\end{array}$ & $\begin{array}{c}\text { Emulsification } \\
\mathbf{a t} \mathbf{0} \mathbf{C}\end{array}$ & $\begin{array}{c}\text { Emulsification } \\
\text { at } \mathbf{5 0} \mathbf{0}^{\circ} \mathbf{C}\end{array}$ \\
\hline $\begin{array}{c}\text { Alcotex 55-002H } \\
\text { Synthomer }\end{array}$ & PVAl55-18 & 55 & $\approx 18$ & Water-in-oil & Water-in-oil \\
\hline $\begin{array}{c}\text { Alcotex B72 } \\
\text { Synthomer }\end{array}$ & PVAl72-52 & 72 & $\approx 52$ & Water-in-oil & Water-in-oil \\
\hline $\begin{array}{c}\text { PVA \#360627 } \\
\text { Sigma-Aldrich }\end{array}$ & PVAl80-10 & 80 & $\approx 10$ & Oil-in-water & Water-in-oil \\
\hline $\begin{array}{c}\text { PVA \#363138 } \\
\text { Sigma-Aldrich }\end{array}$ & PVAl98-50 & $98-99$ & $31-50$ & Oil-in-water & Oil-in-water \\
\hline
\end{tabular}




\section{SI.3: Determination of PVAl80-10 microstructure by NMR}

Degree of hydrolysis

From the ${ }^{1} \mathrm{H}$ NMR spectrum, Fig. S1, it is possible to determine the relative mol \% of hydrolyzed poly(vinyl acetate) units, by fixing at 3 the $3 \mathrm{H}$ of the $\mathrm{CH}_{3}$ acetate signal at $1.9 \mathrm{ppm}$ and integrating the $\mathrm{CH}_{2}$ area of all units from 1.1 to $1.9 \mathrm{ppm}$. The relative mol \% poly(vinyl acetate) and poly(vinyl alcohol) is found equal to $21 \%$ and $79 \%$, respectively.

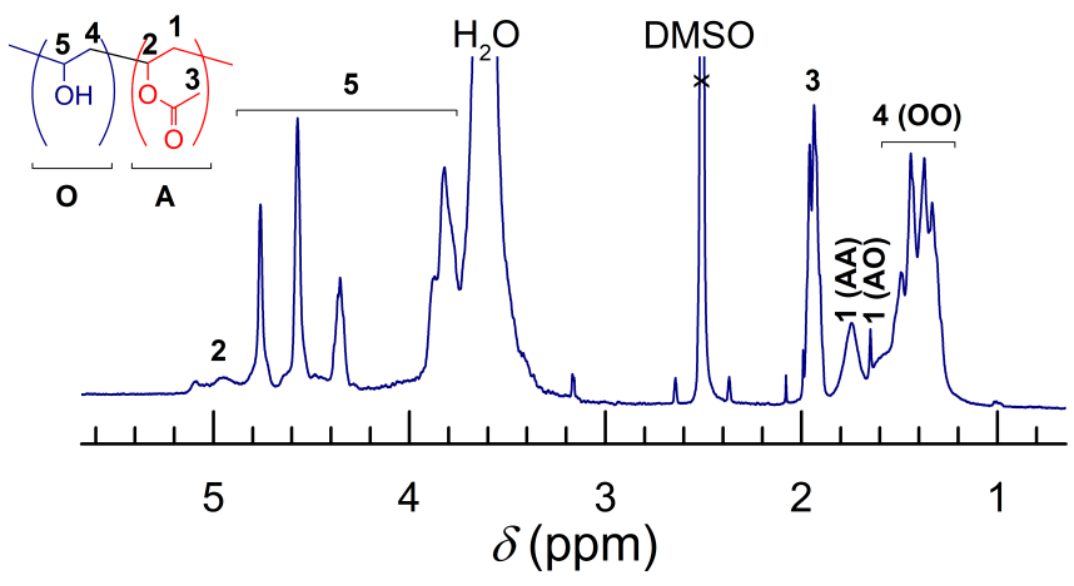

Fig. S1. ${ }^{1} \mathrm{H}$ NMR spectrum of PVA in DMSO-d6.

Even if in the literature, some authors ${ }^{1}$ mentioned using preferably ${ }^{1} \mathrm{H}$ NMR for degree of blockiness calculation, mainly for its higher resolution provided, integration of the involved signals (protons 1 $\mathrm{AA}, 1 \mathrm{AO}$ and $4 \mathrm{OO}$ ) does not seem appropriate and could lead to higher uncertainty. Then calculations of average sequence length and degree of blockiness have been performed in this study by quantitative ${ }^{13} \mathrm{C}$ NMR spectrum, Fig. S2.

From the ${ }^{13} \mathrm{C}$ NMR spectrum, we can clearly distinguish four main areas of signals:

- at about 19 ppm, corresponding to the $\mathrm{CH}_{3}$ of the poly(vinyl acetate) units $\left(\mathrm{C}_{3}\right)$,

- between 37 and 46 ppm, corresponding to the $\mathrm{CH}_{2}$ of both poly(vinyl acetate) $\left(\mathrm{C}_{1}\right)$ and poly(vinyl alcohol) units $\left(\mathrm{C}_{4}\right)$,

- between 63 and 68 ppm, corresponding to the $\mathrm{CH}$ of both poly(vinyl acetate) $\left(\mathrm{C}_{2}\right)$ and poly(vinyl alcohol) units $\left(\mathrm{C}_{5}\right)$,

- from 172 to 174 ppm, corresponding to the $\mathrm{C}=\mathrm{O}$ of the poly vinyl acetate units $\left(\mathrm{C}_{6}\right)$.

As observed in the zoom of the ${ }^{13} \mathrm{C}$ NMR spectrum presented below, the methylene area consists of three well-resolved main groups of signals from 37 to $40 \mathrm{ppm}, 40$ to $42.5 \mathrm{ppm}$ and then 42.5 to $46 \mathrm{ppm}$, that can be assigned to the three following dyad sequences: acetate-acetate (AA), acetatealcohol (AO) and alcohol-alcohol (OO), respectively.

It is then easy to determine the mole ratio of these dyad sequences ( $A A, A O$ and $O O$ ), found equal to $13.2,13.1$ and $73.7 \%$, respectively, and a posteriori to determine the relative molar ratio poly(vinyl acetate) (A) / poly(vinyl alcohol) (O), found equal in percentages to 19.7/80.3 (in good agreement with the one estimated from the ${ }^{1} \mathrm{H}$ NMR spectrum). 
(a)
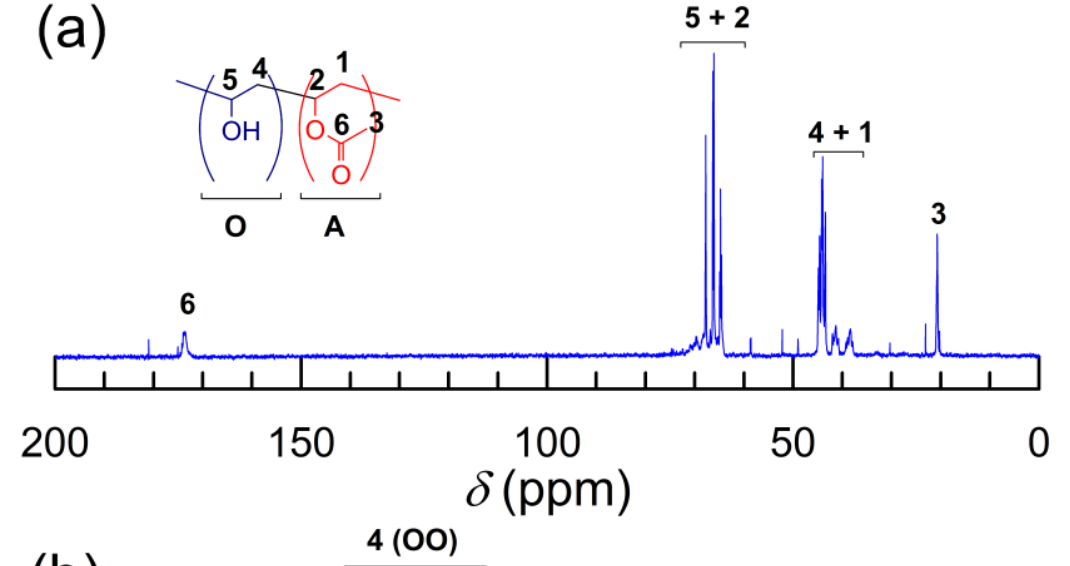

(b) 4 (00)

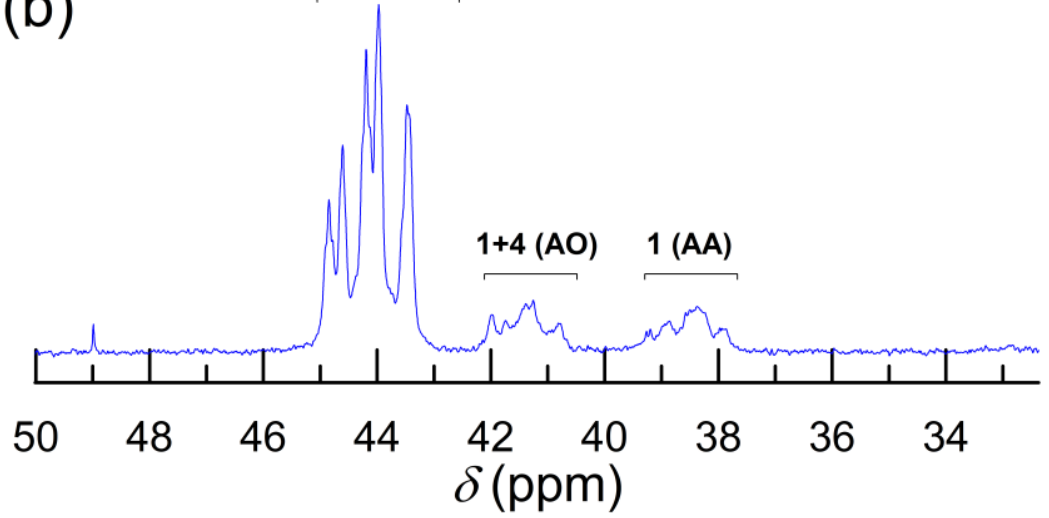

(c)
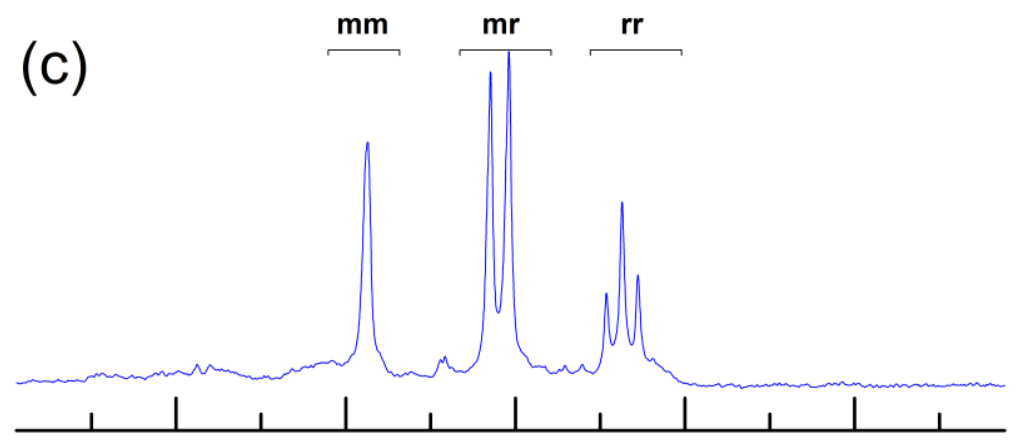

70

68

66

64

$\delta(\mathrm{ppm})$

Fig. S2. Quantitative ${ }^{13} \mathrm{C}$ NMR spectrum of $P V A$ in $\mathrm{D}_{2} \mathrm{O}$ with different spectral ranges of interest.

\section{Blockiness index}

From these different dyad relative areas, it is also possible to determine the degree of blockiness of the PVA sample; high relative molar \% of OO dyads corresponds to a longer mean run length of vinyl alcohol units, while a large amount of $A O$ dyads reveals a more random or alternating sequence distribution.

Considering a quantitative ${ }^{13} \mathrm{C}$ NMR sequence, and that the Nuclear Overhauser Effect is the same for all type of carbons in the polymer backbone, it is possible to apply the following equation to determine the average length of vinyl acetate chains $\left(L_{A C}\right)$, as well as the average length of vinyl alcohol ones $\left(L_{\mathrm{OH}}\right)$ and the degree of blockiness, $\eta$, of the PVA sample. ${ }^{1}$ 


$$
L_{A C}=2 \frac{C_{1}}{C_{1+4}} \quad L_{O H}=2 \frac{C_{4}}{C_{1+4}} \quad \eta=\frac{C_{1+4}}{2 \times C_{4} \times C_{1}}
$$

Applying these equations to the PVA sample, it has been found: $L_{O H}=12.3$ monomers, $L_{O A c}=3.0$ monomers, and $\eta=0.414$. A blockiness index of $0 \leq \eta<1$ is characteristic of block copolymers, $\eta$ $=1$ is for random copolymers and the comonomer tends to alternate in the polymer structure if $1<$ $\eta \leq 2$. Based on this statement, the obtained results tend to show the blocky character of the considered PVA sample.

\section{Tacticity}

As observed on the spectrum above, the methine carbon is nicely split into three well-resolved resonance lines, which have been assigned to the isotactic, heterotactic and syndiotactic triads, annotated $\mathrm{mm}, \mathrm{mr}$ and $\mathrm{rr}$, respectively. The relative areas of these methine carbon triads can be used to determine the tacticity of the polymers. It has been shown in the literature that a single parameter $P_{m}$ can be used to describe the stereochemical configurational sequence of the polymer. ${ }^{2}$ This parameter is defined as the probability that a growing polymer chain forms a sequence with the same relative configuration. The value of $P_{m}$ is obtained from the following formula:

$$
P_{m}=\sqrt{m m}
$$

where $\mathrm{mm}$ is the fraction of isotactic triads (the square root dependency arises from the fact that two successive meso configuration are needed to produce an $\mathrm{mm}$ triad). Values of 1,0 and 0.5 correspond to isotactic, syndiotactic and atactic polymers, respectively.

For the considered sample, $\mathrm{mm}, \mathrm{mr}$ and $r r$ relative molar ratio has been found equal to $0.259,0.497$ and 0.254 , respectively, so $P_{m}$ has been estimated at 0.51 , showing that the polymer is atactic. 


\section{SI.4: Analysis of some PVAl80-10 properties by DSC}

The residual water content of the PVA powder was calculated from the integration of the first (1) endothermic signal during the first run $(102 \mathrm{~J} / \mathrm{g})$. The DSC crystallinity value was calculated using the second (2) endothermic signal (first run). The DSC volumetric degree of crystallinity, $X_{c}$, was calculated using the following equation:

$$
X_{c}(D S C)=\frac{\Delta H}{\Delta H_{c}}=40 \%
$$

where $\Delta H=1.8 \mathrm{~kJ} /$ eq. $\mathrm{OH}$ is the measured enthalpy (taking $192 \mathrm{eq} . \mathrm{OH} / \mathrm{mol} \mathrm{PVA}, M_{n}=8980 \mathrm{~g} / \mathrm{mol}$ and corrected with water content $=4.5 \mathrm{wt} \%$ ) and $\Delta H_{c}=4.39 \mathrm{~kJ} / \mathrm{eq} . \mathrm{OH}$ is the theoretical enthalpy of a fully crystalline PVA. ${ }^{3}$

Glass transition temperature was evaluated on the third run, after water evaporation during first run and cancelation of thermal history during the second run, so as to minimize enthalpic relaxation associated with the glass transition. Its value was estimated at $65^{\circ} \mathrm{C}$ under a dry state.

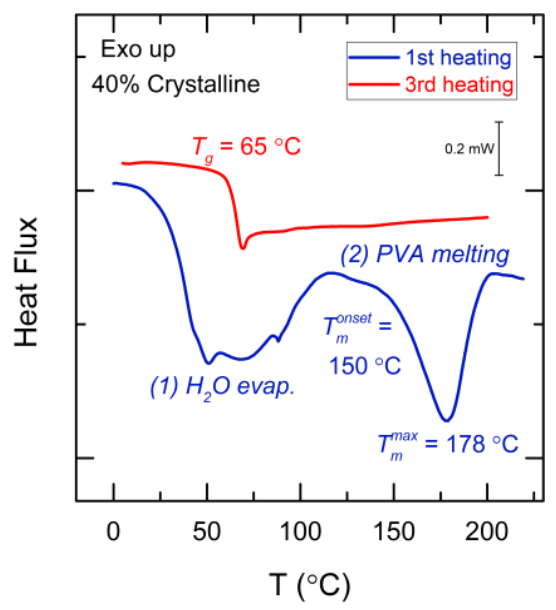

Fig. S3. DSC thermograms of PVA recorded at $3 \mathrm{~K} / \mathrm{min}$. 


\section{SI.5: PVAl80-10 crystallinity determined by X-ray diffraction (XRD)}

The XRD degree of crystallinity of PVA powder was calculated using the areas ratio of all crystalline and amorphous peaks:

$$
X_{c}(X R D)=\frac{\Sigma A_{\text {cryst }}}{A_{\text {amorph }}}=34 \%
$$

A w/o dispersion of $5 \%$ PVAl80-10 (0.1 M sodium sulfate):chloroform (50:50) was also analyzed by XRD.
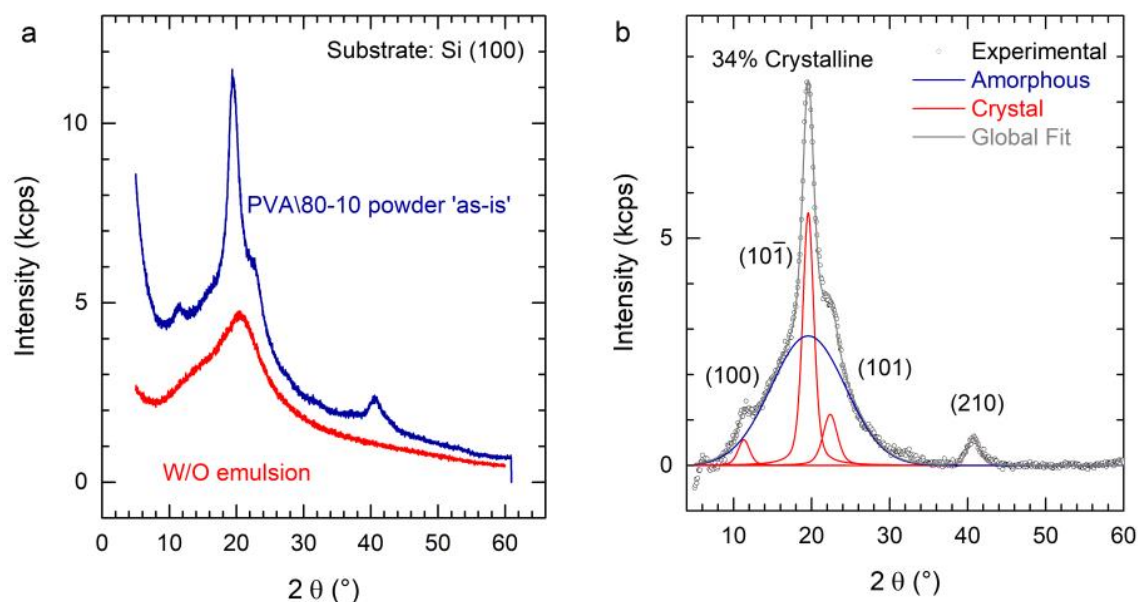

Fig. S4. (a) XRD pattern of a PVA powder and w/o emulsion. (b) Peak deconvolution after baseline subtraction of PVA powder signal. 


\section{SI.6: Determination of PVAl80-10's LCST by DLS}

Fig. S5 shows the evolutions of diameter and PDI as a function of temperature. For PVA concentration $>5 \% \mathrm{w} / \mathrm{v}$, a sharp increase of the characteristic diameter is associated with the cloud point, i.e. aggregation of precipitated polymer coils. For concentrations $\geq 5 \% \mathrm{w} / \mathrm{v}$ the increase is, at first sight, smoother and thus it is difficult to determine the cloud point from $d v s$. $T$ curves. In fact, the experimental cloud point found by visual inspection matches better with the increase of PDI. Thus, for these reasons, we used PDI vs. T curves to determine the cloud points (marked by dotted line in Fig. S5).

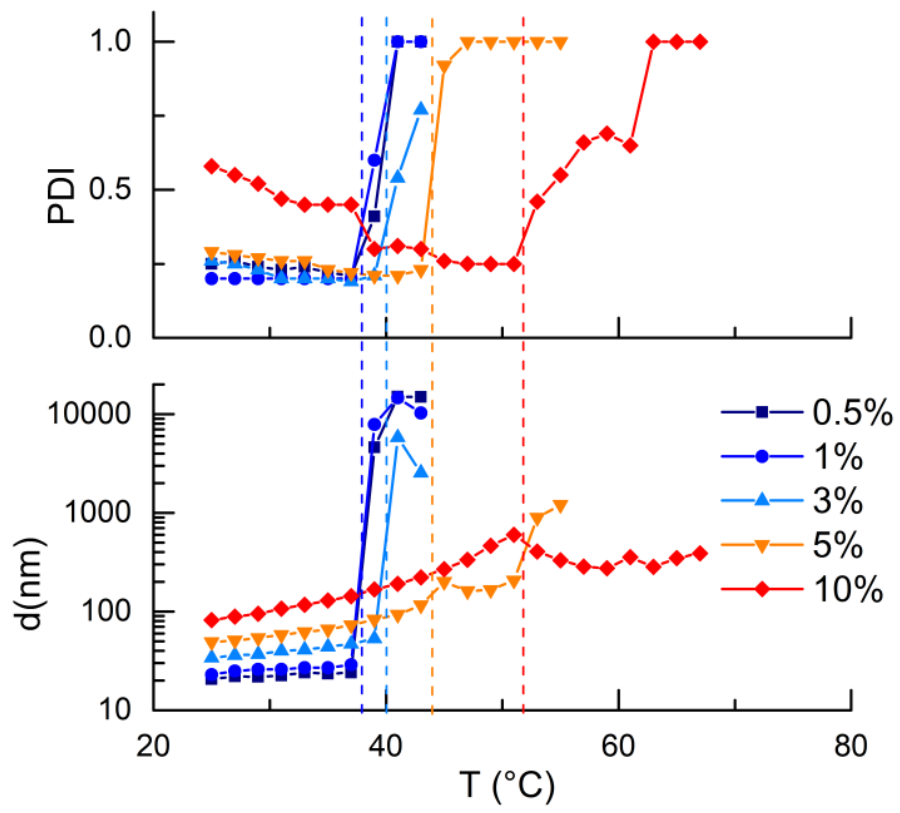

Fig. S5. PDI and characteristic object diameter versus temperature at various PVA concentration, as measured by DLS. Lines are only guides for the eye. 


\section{SI.7: Water-in-chloroform interfacial tensions}

PVAl80-10 yields a significant decrease of the interfacial tension between water $(0.1 \mathrm{M}$ sodium sulfate) and chloroform from $32.8 \mathrm{mN} \mathrm{m}^{-1}$ for pure water-chloroform ${ }^{4}$ to $1.5-2.7 \mathrm{mN} \mathrm{m}^{-1}$. The interfacial tension decreases with temperature and an abrupt drop is observed at LCST, because of polymer precipitation (here estimated around $35^{\circ} \mathrm{C}$ ).

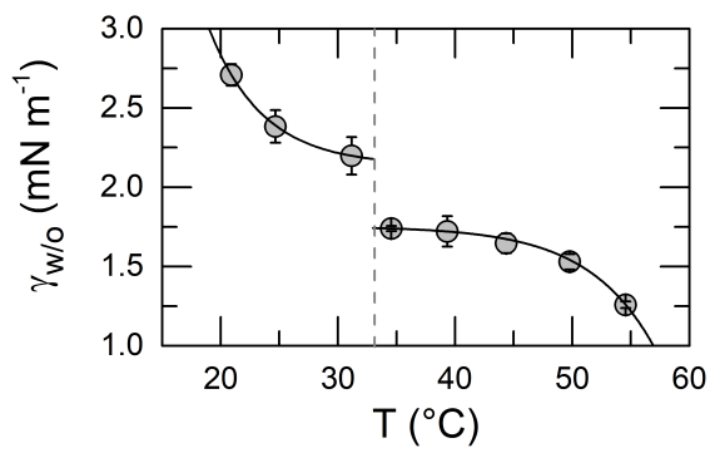

Fig. S6. Interfacial tension as a function of the temperature, between chloroform and an aqueous solution of PVAl80-10 5\% w/v and $0.1 \mathrm{M}$ sodium sulfate. 


\section{SI.8: Histograms of microcapsules}

Image analyses were carried out using ImageJ software. Particle distribution analyses were performed by thresholding the raw images. The capsules were counted when presented a circularity of 0.60-1.40. Microcapsules in contact were therefore mostly omitted. Particle diameter (d) distributions were fit with a log normal function, as:

$$
f(d)=\frac{1}{\sqrt{2 \pi} \cdot \sigma \cdot d} \exp \left(-\frac{\ln \left(\frac{d}{d_{m}}\right)^{2}}{2 \sigma^{2}}\right)
$$

where $d_{m}$ is the geometrical mean diameter and $\sigma$ is the standard deviation of $\ln \left(d_{m}\right)$. The polydispersity index (PDI) was calculated using the following expression ${ }^{5}$ :

$$
\mathrm{PDI}=\exp \left(\sigma^{2}\right)-1
$$
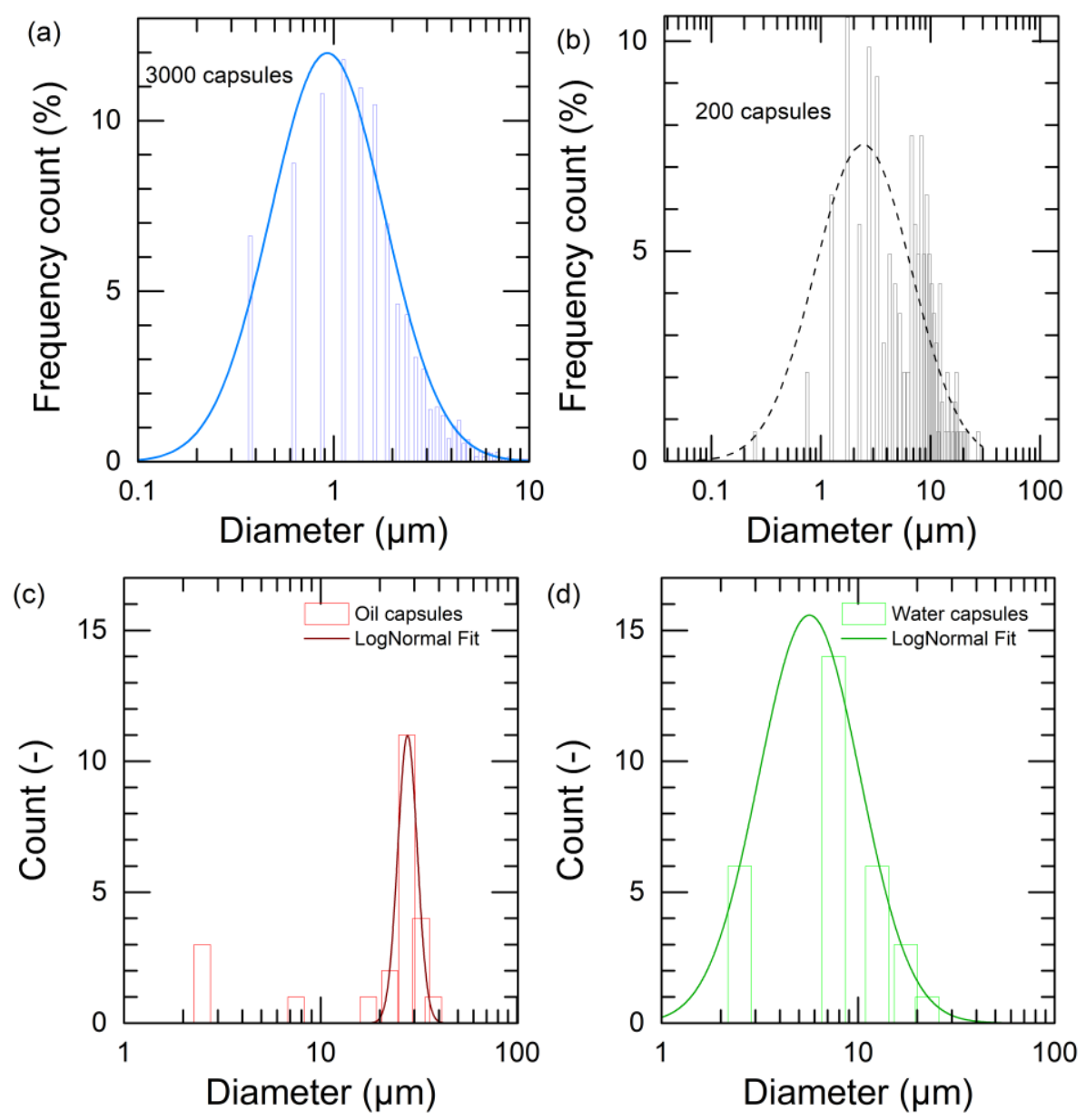
(e)
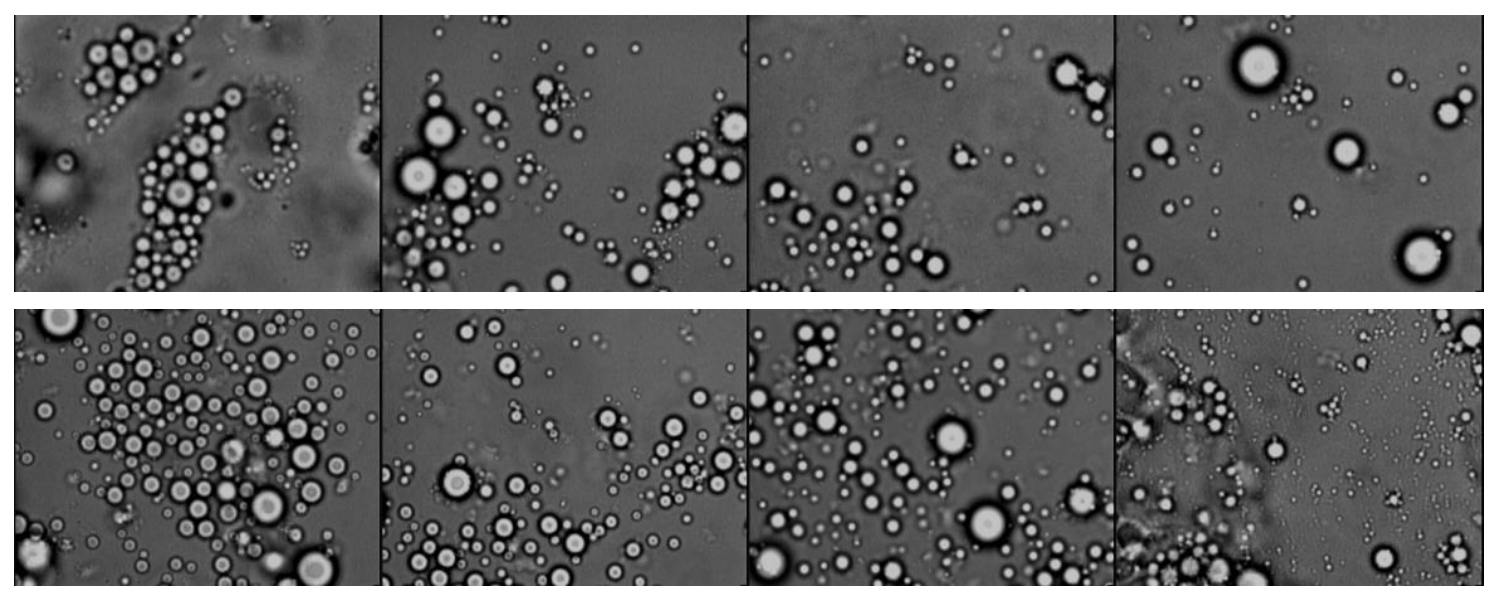

Fig. S7. Typical diameter distributions of capsules using image analysis. (a) according distribution of the images in Fig 3a, (b) according distribution for Fig. 3d, (c) and (d) according distribution for Fig. 4bc. (e) Optical microscopy images corresponding the sample presented in Fig. 3a (scale: $65 \mu \mathrm{m}$-wide image). 


\section{SI.9: Shell thickness of $w / w$ dispersion}

The shell thickness was estimated by plotting the intensity profile of ten capsules (whose shells were made of fluorophore tagged-PVAl80-10, cf. Fig. 3d of the main manuscript). The intensity peaks were fit with Gaussian function and the shell thickness (vide infra) was estimated at the full width at half maximum (FWHM) of the Gaussian fit. Owing to the fact that fluorescent confocal microscopy is diffraction-limited (near $0.8 \mu \mathrm{m}$ ), these values represent only estimations of the true shell thickness.

Table S3. Shell thickness measurements, results are FWHM of Gaussian fits.

\begin{tabular}{|c|c|c|}
\hline Diameter & FWHM 1 & FWHM 2 \\
\hline 5.1 & 0.74 & 0.52 \\
\hline 5.3 & 1.00 & 0.80 \\
\hline 5.6 & 0.62 & 0.90 \\
\hline 5.6 & 0.80 & 0.60 \\
\hline 7.1 & 0.80 & 0.62 \\
\hline 7.4 & 1.12 & 1.00 \\
\hline 10.4 & 0.88 & 0.90 \\
\hline 11.2 & 1.00 & 0.82 \\
\hline 12.1 & 0.82 & 1.08 \\
\hline 13.6 & 0.60 & 0.74 \\
\hline
\end{tabular}




\section{SI.10: Kinetics and optimization of crosslinking reaction}

The conversation of glutaraldehyde vs. time values were calculated using integration of $\mathrm{CHO}$ proton at $9.67 \mathrm{ppm}$ with respect to $\mathrm{CH}_{3}$ protons of internal standard (toluene) at $2.25 \mathrm{ppm}$.
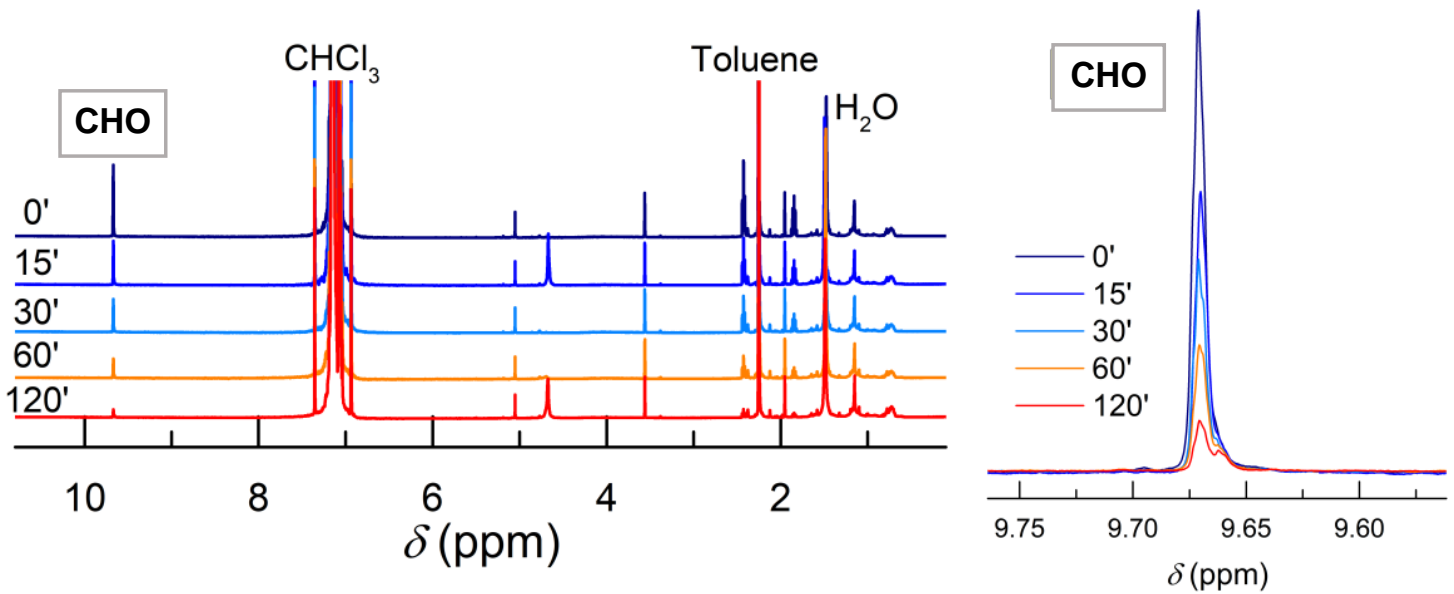

Fig. S8. ${ }^{1} \mathrm{H}$ NMR spectra following the kinetics of capsule crosslinking with glutaraldehyde (in $\left.\mathrm{CDCl}_{3}\right)$.

The molar amount of crosslinker was then optimized to avoid capsule aggregation. For that, PVA $5 \% \mathrm{w} / \mathrm{v} 0.1 \mathrm{M}$ sodium sulfate aqueous solution was allowed to react with different amounts of glutaraldehyde at $\mathrm{pH} 1.3$ and $50{ }^{\circ} \mathrm{C}$, for 2 hours under magnetic agitation. The optimized amount was found at ca. $2 \mathrm{~mol} \%$, ensuring efficient crosslinking without unwanted formation of tightly crosslinked networks that yield interparticle crosslinking.

Table S4. Optimization of glutaraldehyde concentration to perform PVA crosslinking.

\begin{tabular}{|c|c|}
\hline $\begin{array}{c}\text { Glutaraldehyde } \\
\text { (mol\% / OH }\end{array}$ & Observations \\
\hline 0.4 & Clear solution at RT \\
\hline 0.8 & Hazy solution at RT \\
\hline 1.6 & 'Brittle' hydrogel \\
\hline 3.2 & 'Stiff' hydrogel \\
\hline 7 & 'Stiff' hydrogel \\
\hline 14 & 'Stiff' hydrogel \\
\hline 30 & 'Stiff' hydrogel \\
\hline 60 & 'Stiff' hydrogel \\
\hline
\end{tabular}




\section{SI.11: Calibration curve of methyl blue}
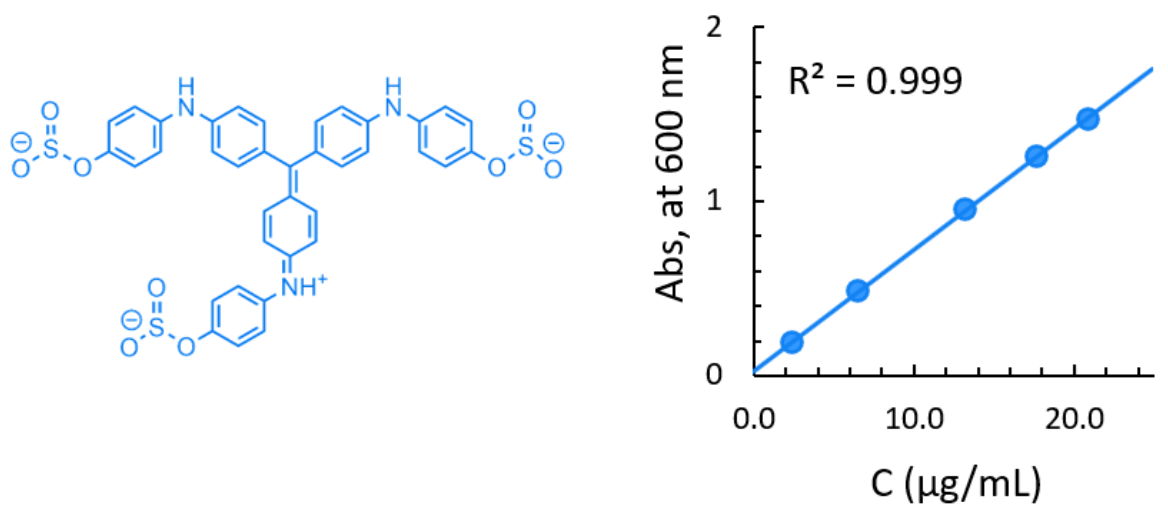

Fig. S9. Methyl blue chemical structure and its UV-vis calibration curve. 


\section{SI.12: Phase diagrams}
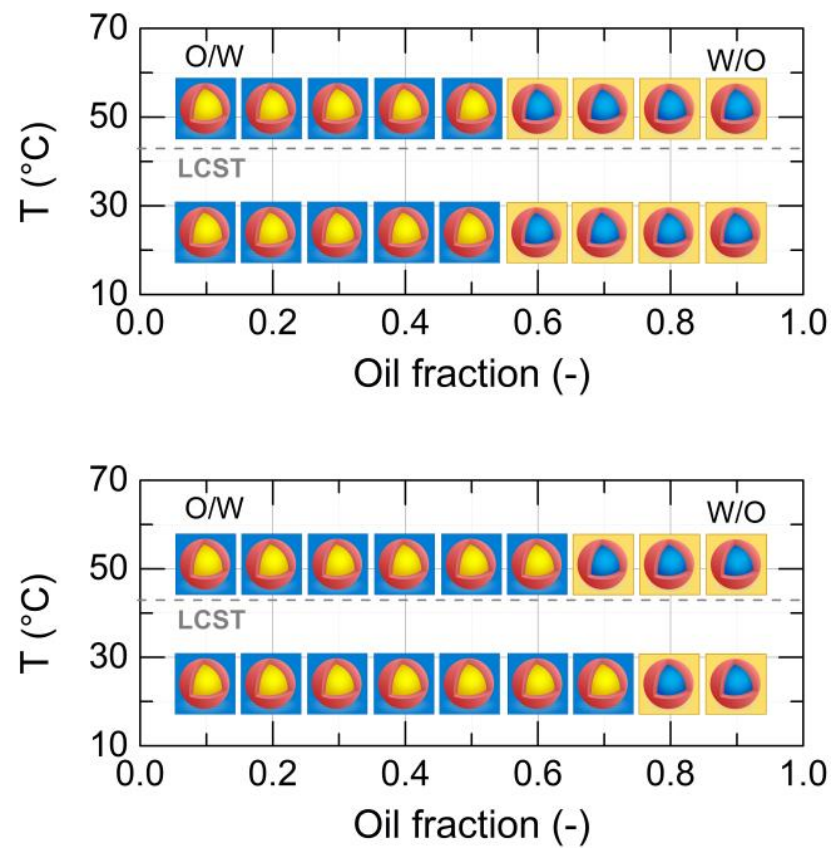

Fig. S10. Phase diagrams showing the type of emulsions as a function of oil content (respectively to PVA $5 \% \mathrm{w} / \mathrm{v}$ in $0.1 \mathrm{M}$ sodium sulfate aqueous solution) and temperature. Grey dashed line represents the LCST. Oil: ethyl acetoacetate (top) and $\alpha$-terpineol (bottom). 


\section{SI.13: Images of ethyl acetoacetate-water dispersions}

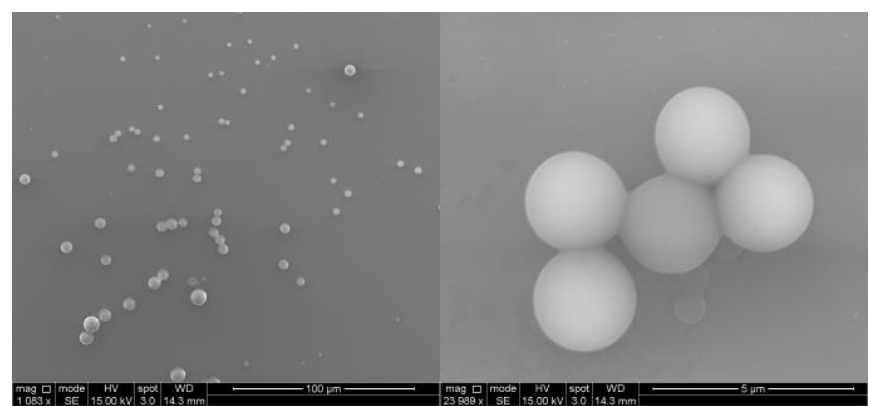

Fig. S11. SEM micrographs of w/o dispersions (oil: ethyl acetoacetate).
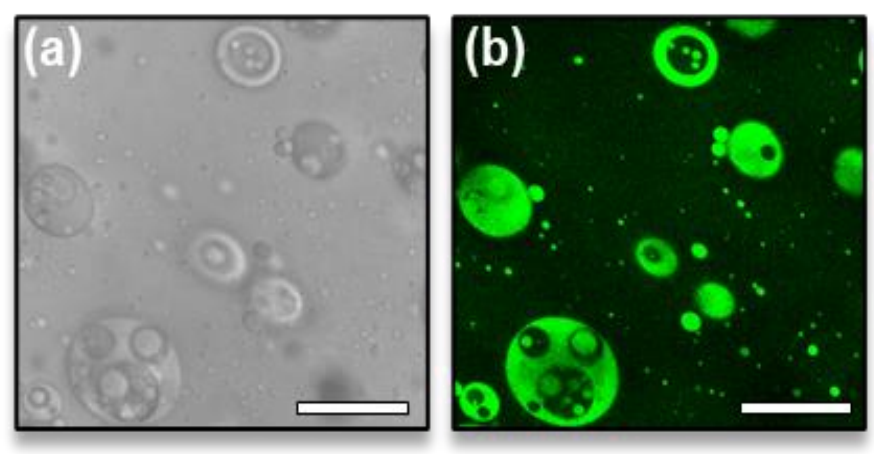

Fig. S12. (a) Transmission confocal microscopy image of a water-in-oil-in-water-in-oil dispersion and (b) its according fluorescence images highlighting hydrophilic domains (Rhodamine $110 \mathrm{HCl}$ ). Scale-bars represent $50 \mu \mathrm{m}$. 


\section{SI.14: Movie description}

The movie intitled "o-w_dispersion-RT.mp4" shows an emulsification process at room temperature, between chloroform and a 5\% w/v PVAl80-10, $0.1 \mathrm{M}$ sodium sulfate, aqueous solution ( $T<\mathrm{LCST}$ ), yielding oil-in-water dispersion by simple hand shaking. The dispersion was then poured into water to verify that the water was the continuous phase.

The movie intitled "w-o_dispersion-50C.mp4" shows an emulsification process between chloroform and a $5 \% \mathrm{w} / \mathrm{v}$ PVAl80-10, $0.1 \mathrm{M}$ sodium sulfate, aqueous solution which were heated at $50{ }^{\circ} \mathrm{C}$ in an oven. Note that the PVA solution is hazy ( $T>$ LCST). The dispersion was then poured into water and the formation of chloroform droplet shows well that it yielded a w/o dispersion. 


\section{References}

(1) Budhlall, B. M.; Landfester, K.; Sudol, E. D.; Dimonie, V. L.; Klein, A.; El-Aasser, M. S. Characterization of Partially Hydrolyzed Poly(Vinyl Alcohol). Effect of Poly(Vinyl Alcohol) Molecular Architecture on Aqueous Phase Conformation. Macromolecules 2003, 36 (25), 9477-9484.

(2) Bugada, D. C.; Rudin, A. Characterization of Poly(Vinyl Alcohol-Acetate) by $13 \mathrm{C}$ n.m.r. and Thermal Analyses. Polymer (Guildf). 1984, 25 (12), 1759-1766.

(3) Peppas, N. A.; Merrill, E. W. Differential Scanning Calorimetry of Crystallized PVA Hydrogels. J. Appl. Polym. Sci. 1976, 20 (6), 1457-1465.

(4) Demond, A. H.; Lindner, A. S. Estimation of Interfacial Tension between Organic Liquids and Water. Environ. Sci. Technol. 1993, 27 (12), 2318-2331.

(5) Thomas, J. C. The Determination of Log Normal Particle Size Distributions by Dynamic Light Scattering. J. Colloid Interface Sci. 1987, $117(1), 187-192$. 